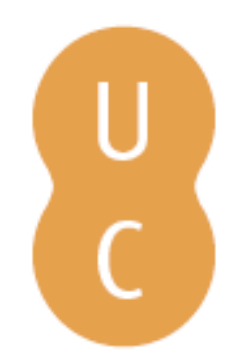

\title{
nommalina
}

\section{Coping Health Inventory for Parents (CHIP)}

Autor(es): $\quad$ Cunha, Ana Isabel; Major, Sofia; Relvas, Ana Paula

Publicado por: Imprensa da Universidade de Coimbra

URL

persistente:

URI:http://hdl.handle.net/10316.2/40591

DOI:

DOI:https://doi.org/10.14195/978-989-26-1268-3_7

Accessed : $\quad$ 26-Apr-2023 15:04:38

A navegação consulta e descarregamento dos títulos inseridos nas Bibliotecas Digitais UC Digitalis, UC Pombalina e UC Impactum, pressupõem a aceitação plena e sem reservas dos Termos e Condições de Uso destas Bibliotecas Digitais, disponíveis em https://digitalis.uc.pt/pt-pt/termos.

Conforme exposto nos referidos Termos e Condições de Uso, o descarregamento de títulos de acesso restrito requer uma licença válida de autorização devendo o utilizador aceder ao(s) documento(s) a partir de um endereço de IP da instituição detentora da supramencionada licença.

Ao utilizador é apenas permitido o descarregamento para uso pessoal, pelo que o emprego do(s) título(s) descarregado(s) para outro fim, designadamente comercial, carece de autorização do respetivo autor ou editor da obra.

Na medida em que todas as obras da UC Digitalis se encontram protegidas pelo Código do Direito de Autor e Direitos Conexos e demais legislação aplicável, toda a cópia, parcial ou total, deste documento, nos casos em que é legalmente admitida, deverá conter ou fazer-se acompanhar por este aviso. 
AVALIAÇÃO

$$
\text { FAMILIAR Vorstil Aorracicio }
$$

Q)

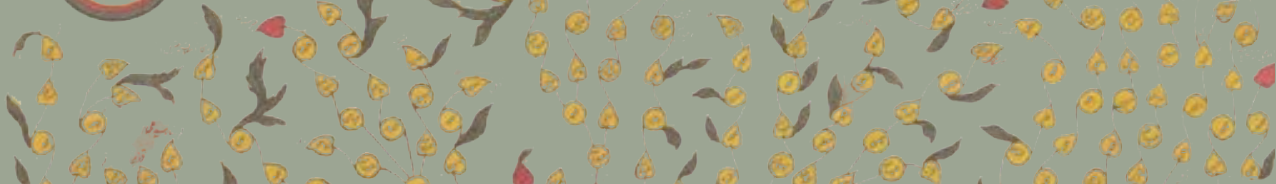

$-1000000$

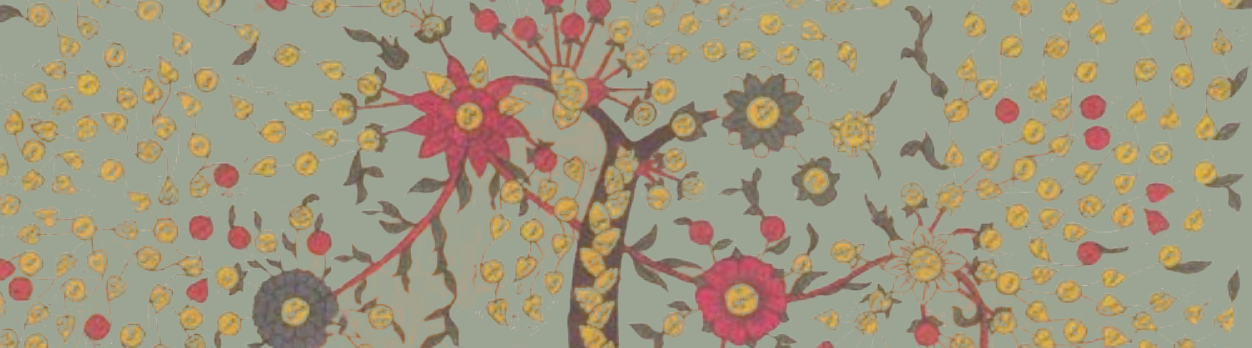

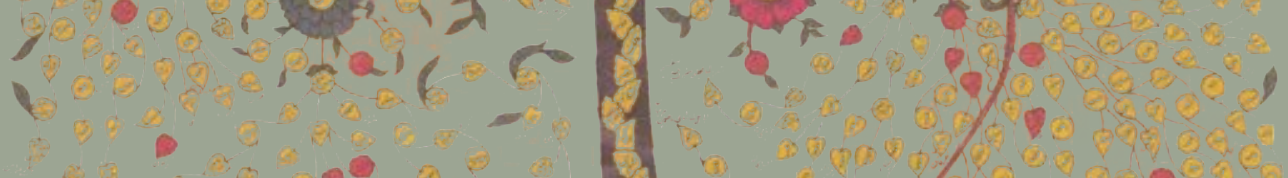

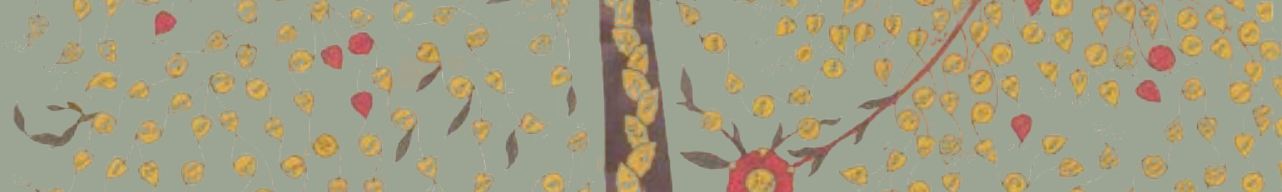

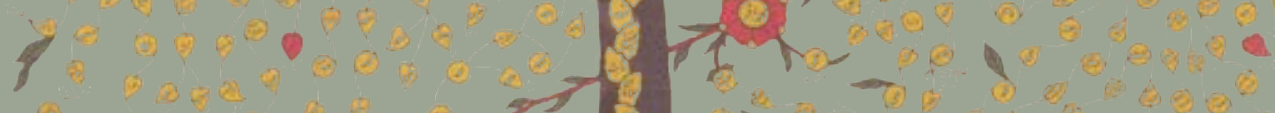

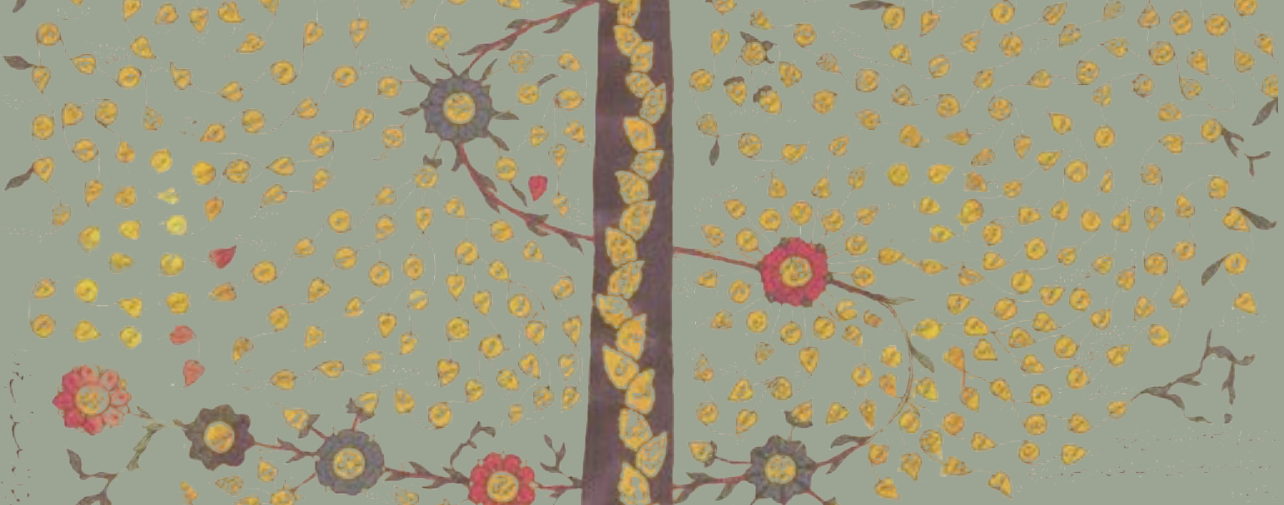

ANA PAULA RELVAS

SOFFA MAJOŔR Wh DE COIMBRA

COORDENAÇÃO 


\title{
COPING HEALTH INVENTORY FOR PARENTS ( C H I P)
}

\author{
Ana Isabel Cunha \\ Sofia Major \\ Ana Paula Relvas
}

"A total family system perspective on coping is needed to view the impact on the family and what steps can be taken to belp the family to adapt."

\section{Resumo}

O Coping Health Inventory for Parents (CHIP; McCubbin et al., 1983) é um inventário composto por 45 itens que permitem avaliar a perceção dos pais sobre as suas respostas de coping, para lidar com a vida familiar, quando têm um filho gravemente e/ou cronicamente doente. Neste capítulo são apresentados os estudos de adaptação e validação da versão portuguesa do CHIP numa amostra de 143 pais e mães de crianças com diferentes doenças crónicas. A análise fatorial exploratória indica-nos uma solução fatorial de três fatores, tal como a versão original, embora sem replicar a solução fatorial original quanto à distribuição dos itens pelos fatores. Os estudos de análise fatorial confirmatória revelam que o modelo original não se adequa aos dados recolhidos. O instrumento apresentou boa consistência 
interna ao nível dos três fatores ( $\alpha$ entre $.80-.88$ ). Os resultados são discutidos tendo em consideração as características e utilidade da versão portuguesa do CHIP.

Palavras-chave: coping parental, doença crónica pediátrica, CHIP, análise fatorial.

\begin{abstract}
The Coping Health Inventory for Parents (CHIP; McCubbin et al., $1983)$ is a 45 item instrument designed to assess parents' appraisal of coping behaviors they are using to manage family life when they have a seriously or chronically ill child. This chapter presents the adaptation and validation studies of the Portuguese version of CHIP in a sample of 143 parents of children with different chronic illnesses. Exploratory factor analysis yielded a three-factor solution, although it did not replicate the three-factor structure of the original CHIP. Confirmatory factor analysis demonstrated that the original model does not fit to the data. The instrument revealed good internal reliability for its factors ( $\alpha$ between $.80-.88$ ). Results are discussed concerning the characteristics and utility of the Portuguese version of CHIP.
\end{abstract}

Keywords: parental coping, childhood chronic illness, CHIP, factor analysis.

\title{
1. Instrumento \\ O que é, o que avalia e a quem se aplica?
}

No Quadro 1 encontra-se a ficha técnica relativa ao Coping Health Inventory for Parents (CHIP; McCubbin, et al., 1983). 
Quadro 1.

Ficha técnica do CHIP

O que é?

A versão portuguesa do CHIP - Coping Health Inventory for Parents, escala publicada originalmente, em 1983, por Hamilton McCubbin e colaboradores

O CHIP é um inventário de auto-resposta que avalia a perceção dos pais sobre as suas respostas de coping, para lidar com a vida familiar quando têm um filho gravemente e/ou cronicamente doente. Contém 45 itens que se distribuem por três subescalas/padrões: Integração Familiar, Cooperação e Definição Otimista da Situação; Manutenção do Suporte Social, Auto-Estima e Estabilidade Psicológica; e Compreensão da Situação Médica através da Comunicação com outros Pais e Consulta com os Membros da Equipa de Saúde

Estrutura do CHIP (versão original)

\begin{tabular}{ccc}
\hline Subescala & $\begin{array}{c}\text { Número } \\
\text { Itens }\end{array}$ & Descrição \\
\hline
\end{tabular}

Integração Familiar, Cooperação e De-

finição Otimista da Situação (Padrão I)
Comportamentos centrados no fortalecimento da vida familiar e das relações e na visão que pais têm sobre a vida familiar

\section{O que avalia?}

Manutenção do Suporte Social, Auto-Estima e Estabilidade Psicológica (Padrão II)
Esforços parentais para manter o seu próprio "bem-estar" através de relações sociais, envolvimento em atividades 18 que aumentem a auto-estima e comportamentos para lidar com as tensões e pressões psicológicas
Compreensão da

Situação Médica através da Comunicação com outros Pais e Consulta com os Membros da Equipa de Saúde (Padrão III)
A quem se aplica?

O CHIP foi desenvolvido para ser utilizado com pais de crianças com condições de saúde graves e/ou crónicas

O acesso à versão portuguesa do CHIP pode ser efetuado através da página http://www.fpce.uc.pt/avaliacaofamiliar que contém todos os instrumentos de avaliação apresentados neste livro. Os utilizadores

Como ter acesso? deverão facultar os contactos pessoais e institucionais, bem como dados acerca do propósito da utilização do instrumento (e.g., investigação, prática clínica) e concordar com as condições de utilização e de partilha dos resultados com os autores da versão portuguesa 


\section{Fundamentação e história}

O conceito de resiliência é, em geral, utilizado para descrever a capacidade de adaptação positiva num contexto de adversidade. Os modelos baseados nas teorias do stress e resiliência familiares foram inovadores ao abordar a adaptação e resiliência a um nível familiar, destacando o papel que propriedades, comportamentos e capacidades familiares desempenham na recuperação das famílias em situações de crise (McCubbin \& McCubbin, 1996).

Neste sentido, a adoção de uma visão sistémica da resiliência permitiu alargar o conceito e aplicá-lo ao funcionamento das famílias, referindo-se o termo resiliência familiar ao "percurso que a família percorre, à medida que se adapta e prospera face ao stress, tanto no presente, como ao longo do tempo" (Hawley \& DeHann, 1996, p. 293). Assim, a abordagem da resiliência familiar desvia-se das perspetivas tradicionais de investigação e intervenção baseadas nos défices, salientando o poder regenerativo das famílias (Walsh, 2002) e considerando que o que distingue famílias saudáveis não é o facto de não apresentarem problemas, mas sim as suas capacidades de coping e de resolução dos mesmos (Walsh, 1998).

O Modelo de Resiliência Familiar - Resiliency Model of Family Adjustment and Adaptation (McCubbin \& McCubbin, 1996) representa uma extensão das teorias de stress familiar, contribuindo para a compreensão dos processos ativos que as famílias utilizam para equilibrar as exigências com as capacidades das famílias, e que as podem conduzir a um nível de ajustamento ou adaptação familiar. Estas teorias consideram o coping como um processo ativo que integra não só a utilização de recursos familiares existentes, mas também o desenvolvimento de novos comportamentos que ajudem a fortalecer a unidade familiar e a reduzir o impacto dos acontecimentos de stress (McCubbin, McCubbin, Nevin, \& Cauble, 1981). Deste modo, o conceito de coping refere-se a estratégias ativas ou passivas, padrões e comportamentos que são desenvolvidos para manter ou fortalecer a unidade familiar, conservar a estabilidade emocional e o bem-estar dos membros da família, procurar ou utilizar recursos familiares 
ou da comunidade e desencadear esforços para resolver as dificuldades criadas pelo acontecimento de stress (McCubbin \& McCubbin, 1996).

Uma doença grave e/ou crónica numa criança reflete-se em toda a família enquanto sistema e é, habitualmente, considerada como uma significativa fonte de stress familiar. Ao trabalhar com famílias com crianças com doenças crónicas, é importante que os profissionais avaliem o coping parental, no sentido de perceber se a adaptação está a ser positiva ou se é necessária uma intervenção no sentido de melhorar as respostas de coping e a vida familiar (McCubbin, Thompson, \& McCubbin, 2001). Foi com o objetivo de criar uma medida que permitisse avaliar a perceção que os pais têm sobre as suas respostas de coping para lidar com a vida familiar, quando têm um filho gravemente e/ou cronicamente doente, que McCubbin e colaboradores (1983) criaram o Coping Health Inventory for Parents (CHIP).

No processo de construção deste instrumento foram incluídos itens referentes a comportamentos de coping, utilizados em estudos prévios sobre respostas de coping familiar ao stress (cf. McCubbin et al., 1981; 1983), mas foram também desenvolvidos itens adicionais baseados, nomeadamente, nas teorias do suporte social, de stress familiar, de coping individual e de suporte nos cuidados de saúde familiares.

A versão original do CHIP era composta por 80 itens e, aquando do seu desenvolvimento, foi aplicada uma abordagem hierárquica da organização dos comportamentos, sendo definidos dois níveis gerais. Um primeiro nível dizia respeito aos comportamentos de coping, que foram operacionalmente definidos por 80 itens. Um segundo nível, representativo dos padrões de coping, resultou da combinação desses itens em padrões específicos (McCubbin et al., 1983). Com base numa amostra de 185 pais de crianças com fibrose quística (95 mães e 90 pais), 35 destes itens iniciais foram eliminados (30 por terem sido considerados pelos pais como "não aplicáveis"; outros cinco pelo critério de variância mínima ou insignificante). Os restantes 45 itens foram sujeitos a uma análise de componentes principais (com rotação varimax). Os resultados apontaram para uma estrutura de três fatores que explicavam $71.1 \%$ da variância total. Estes fatores passaram a designar-se "padrões de coping", com valores de 
consistência interna (coeficiente alfa de Cronbach) de .79 para o Padrão I, .79 para o Padrão II e .71 para o Padrão III (McCubbin et al., 1983). Assim, o Padrão I (Integração Familiar, Cooperação e Definição Otimista da Situação) é composto por 19 itens que se focalizam no fortalecimento da vida familiar e das relações e na visão que pais têm sobre a vida familiar com uma criança com uma doença crónica (e.g., item 1 "Conversar, com o cônjuge/companheiro(a), sobre sentimentos pessoais e preocupações”). O Padrão II (Manutenção do Suporte Social, Auto-Estima e Estabilidade Psicológica) inclui 18 itens que representam os esforços parentais no desenvolvimento de relações com outros, na realização de atividades que promovam sentimentos de identidade e valor individual, e comportamentos para lidar com as tensões e pressões psicológicas (e.g., item 2 "Estabelecer relacionamentos e amizades que me ajudem a sentir-me importante e apreciado"). Por fim, o Padrão III (Compreensão da Situação de Cuidados de Saúde através da Comunicação com outros Pais e Consulta com os Membros da Equipa de Saúde) é formado por oito itens centrados no relacionamento parental com a equipa de profissionais de saúde e com outros pais de crianças com doenças crónicas, incluindo comportamentos como o desenvolvimento de maior conhecimento e compreensão sobre a doença e o controlo de cuidados ambulatórios e regimes médicos prescritos (e.g., item 10 "Falar com outras pessoas/ pais na mesma situação que eu”) (McCubbin et al., 1981). Cada um dos 45 itens é cotado numa escala de Likert com quatro níveis de resposta ( 0 = "Nada útil"; 1 = "Minimamente útil"; 2 = "Útil"; 3 = "Extremamente útil”). Existe ainda a possibilidade de indicar a não utilização de um comportamento, assinalando a razão (porque escolheram não o fazer ou porque não é possível na sua família). Os autores disponibilizam uma versão do instrumento em inglês e outra em espanhol.

Os primeiros estudos de evidência de validade do CHIP foram realizados através de uma análise discriminante entre famílias de crianças com paralisia cerebral, com alto e baixo nível de conflito familiar, tendo sido encontradas pontuações significativamente mais elevadas na utilização destas estratégias de coping pelas mães e pelos pais nas famílias com alto conflito, quando comparadas com as de baixo conflito (cf. McCubbin 
et al., 2001). Se aparentemente estes resultados parecem contrários ao que seria de esperar (i.e. utilização de estratégias de coping associadas a baixo conflito), os autores consideram que são, no entanto, consistentes do ponto de vista teórico, uma vez que demonstram que os comportamentos de coping se desenvolvem como resposta a situações de stress, sendo o conflito elevado numa família um índice de stress familiar. No estudo com famílias de crianças com fibrose quística (McCubbin et al., 1983), foi avaliada a validade dos três padrões de coping, utilizando uma medida de funcionamento familiar (Family Environment Scale, FES; Moos, 1974) e dois índices do estado de saúde da criança (índice altura/peso e índice de funcionamento pulmonar). No caso das mães, os Padrões de coping I e III revelaram estar positivamente associados à coesão familiar e o Padrão II à expressividade (avaliadas pela FES). No caso dos pais, o Padrão I demonstrou estar positivamente associado à coesão e organização familiares e negativamente relacionado com o conflito. Verificou-se, também, uma associação positiva entre a organização e controlo familiares e a utilização do Padrão III pelos pais. Foram ainda encontradas associações estatisticamente significativas entre padrões de coping maternos e paternos e melhoras no estado de saúde das crianças, refletidas nos índices de altura/peso e de funcionamento pulmonar.

O CHIP tem sido largamente utilizado em investigações com pais de crianças com uma grande diversidade de condições/doenças crónicas, nomeadamente diabetes mellitus tipo I (Auslander, Bubb, Rogge, \& Santiago, 1993; Stallwood, 2005), asma (Garro, 2011), cancro (Goldbeck, 2001; Han, Cho, Kim, \& Kim, 2009), artrite idiopática juvenil (Cavallo et al., 2009), fibrose quística (Patterson, Budd, Goetz, \& Warwick, 1993), epilepsia (Mu, 2005) e infeção por VIH (Lesar \& Maldonado, 1996). Em Portugal, Pereira, Almeida, Rocha e Leandro (2011) utilizaram uma versão de investigação deste instrumento para avaliar as respostas de coping de pais de adolescentes com diabetes mellitus tipo I. A maioria das investigações que utilizam este instrumento examina os padrões de coping parental em diferentes grupos de doenças, mantendo a estrutura fatorial originalmente proposta pelos autores, sendo, em alguns casos, analisados os valores da consistência interna dos itens. No entanto, 
procurando contribuir para o estudo do instrumento num contexto social e cultural distinto, Aguilar-Vafaie (2008) examinou a estrutura fatorial do CHIP numa amostra de 75 mães e pais Iranianos de crianças com cancro. Os resultados apontaram para a existência de três fatores (i.e. Integração Familiar, Fortalecimento do Self e Compreensão da Situação Médica), numa versão adaptada do CHIP com 13 itens, sugerindo um espectro mais reduzido de comportamentos de coping parental, em termos de número e variedade, neste contexto específico.

\section{Estudos em Portugal}

Como foi desenvolvido/adaptado e validado?

\section{Estudos de tradução e adaptação}

Os estudos de tradução e adaptação do CHIP para português fazem parte de um processo decorrido entre 2008 e $2013^{6}$. Num primeiro momento, contactaram-se os autores da versão original, no sentido de obter permissão para traduzir e utilizar o CHIP. Uma vez obtida a autorização, os itens foram traduzidos para a língua portuguesa, procurando manter-se o mais possível o seu sentido original. Posteriormente, foi realizada uma retroversão independente, para a língua inglesa, por um professor com formação em filologia germânica. Procedeu-se à comparação das versões em inglês e em português e realizaram-se alguns ajustes no sentido de alcançar uma versão consensual. Dada a existência de dúvidas relativas à interpretação do significado de um item em particular (item 7), contactaram-se os autores que prontamente esclareceram o significado do referido item, ultimando-se assim a versão final do instrumento (CHIP; Tradução portuguesa: Cunha \& Relvas, 2008).

\footnotetext{
${ }^{6}$ Projeto desenvolvido no âmbito de uma investigação de Doutoramento em Psicologia Clínica (Cunha, 2011), na Faculdade de Psicologia e de Ciências da Educação da Universidade de Coimbra, com bolsa de doutoramento da Fundação para a Ciência e Tecnologia (SFRH/BD/38022/2007).
} 
De seguida, realizou-se um estudo piloto com o CHIP junto de familiares de crianças com doença crónicas, no sentido de avaliar a compreensibilidade dos itens, da escala de cotação e instruções, e averiguar possíveis dificuldades de interpretação. De um modo geral, não se verificaram quaisquer dúvidas em relação ao seu preenchimento.

Concluído este processo, procedeu-se à administração do CHIP a um conjunto de pais e mães de crianças com diferentes doenças crónicas, no âmbito de um estudo mais alargado sobre adaptação familiar à doença crónica pediátrica (Cunha, 2011). Neste sentido, o CHIP integrava um protocolo composto por outros questionários familiares, para além de um questionário de dados sociodemográficos, familiares e sobre a doença ${ }^{7}$. Os dados foram recolhidos em dois hospitais públicos e em centros de saúde da região centro. O pedido de colaboração às instituições, acompanhado do projeto de investigação detalhado, foi sujeito à apreciação das Comissões de Ética e autorizado pelos respetivos Conselhos de Administração e pelo Conselho Diretivo da Administração Regional de Saúde do Centro.

O processo de recolha de dados ocorreu em duas fases. Num primeiro momento, o recrutamento da amostra foi efetuado apenas em contexto hospitalar, nas consultas de especialidade. A seleção dos participantes incluiu, como critério, ser pai ou mãe de uma criança com diagnóstico de doença crónica. As condições para o acesso a uma amostra de estudo, facultadas pelas instituições hospitalares, conduziram a que os participantes fossem pais de crianças com os seguintes diagnósticos médicos: asma, diabetes mellitus tipo I e artrite idiopática juvenil. O contacto com os participantes foi feito através dos médicos das consultas de especialidade, tendo os pais das crianças sido convidados a participar no estudo no dia da consulta médica dos filhos. Em situações em que ambos os pais acompanhavam a criança, foi solicitada a participação do casal (preenchimento independente). Os participantes foram devidamente informados sobre os objetivos e metodologia do estudo, foi garantido

7 O processo de recolha de dados referentes ao CHIP foi simultâneo ao do FHI (Family Hardiness Index), cuja descrição se encontra no capítulo 3. 
o anonimato e confidencialidade das respostas, o carácter voluntário da participação e assegurado, através do preenchimento de um documento, o seu consentimento informado para participar no estudo. A maioria dos participantes completou o protocolo no contexto hospitalar, numa sala reservada para o efeito (antes ou depois da consulta médica da criança). O questionário de dados sociodemográficos, familiares e sobre a doença foi preenchido na presença da investigadora, em formato de entrevista. Nesta fase de recrutamento da amostra, foram recolhidos dados de 88 mães e pais de 72 crianças com as referidas doenças crónicas.

Numa segunda fase, a amostra foi alargada com a integração de mais 56 mães e pais de crianças com os mesmos diagnósticos médicos, seguidas nas mesmas instituições hospitalares e também em centros de saúde da região centro. Este grupo de pais fez também parte de um estudo de natureza qualitativa que incluía, para além do protocolo de questionários familiares, uma entrevista familiar sobre a experiência de viver com uma criança com uma doença crónica (Cunha, 2011). Neste grupo, foram definidos como critérios de inclusão na amostra: a) estarem presentes as duas figuras parentais na entrevista; b) idade atual da criança $\geq 2$ anos e $\leq 12$ anos; e c) condição diagnosticada no mínimo há um ano e no máximo há seis anos. Assegurou-se o cumprimento dos procedimentos éticos anteriormente referidos e o contacto com os participantes foi, igualmente, feito através dos médicos das consultas de especialidade. No entanto, a condução da entrevista (e preenchimento do protocolo) foi, na sua grande maioria, agendada para um momento posterior e realizada fora das instituições de saúde (e.g., casa das famílias). O protocolo composto pelos questionários familiares foi preenchido por ambos os pais, separadamente, após a realização da entrevista familiar.

Após análise das não-respostas (missing values), um sujeito foi retirado da amostra por não responder a 16 dos 45 itens do CHIP. Assim, a amostra total integra 143 pais de crianças com diabetes mellitus tipo I ( $n=68)$, asma $(n=61)$ e artrite idiopática juvenil $(n=14)$. As mães $(n$ =93) apresentam como idade média 36.96 anos $(D P=5.27)$, variando entre os 22 e os 53 anos, e os pais $(n=50)$ uma média de idades de 38.86 anos $(D P=5.35)$, com idades compreendidas entre os 30 e os 54 
anos. No que respeita ao nível de escolaridade das mães, verificou-se que a frequência ou conclusão do ensino secundário (31.3\%) foi o nível mais representado, seguido da frequência ou conclusão do ensino pós-secundário ou superior (27\%). Relativamente aos pais, observou-se uma maior representação da frequência ou conclusão do ensino secundário (36\%), seguido da frequência ou conclusão do $3^{\circ}$ ciclo do ensino básico (34\%). Em relação à categoria profissional, e seguindo a classificação nacional das profissões do Instituto Nacional de Estatística (2010), verificou-se que, no caso das mães, profissões enquadradas no nível 2 (Especialistas das atividades intelectuais e científicas) foram as mais frequentes (22.8\%), seguidas das profissões de nível 5 (Trabalhadores dos serviços pessoais, de proteção e segurança e vendedores), com 16.1\%, e de nível 4 (Pessoal administrativo), com 14.0\%. De notar que $12.9 \%$ das mães referiram como ocupação ser "doméstica" e que $10.8 \%$ se encontravam desempregadas. Quanto aos pais, a categoria profissional mais representada foi do nível 8 (Operadores de instalações e máquinas e trabalhadores da montagem), com $24.0 \%$, seguida do nível 5 (Trabalhadores dos serviços pessoais, de proteção e segurança e vendedores), com $22.0 \%$, e do nível 7 (Trabalhadores qualificados da indústria, construção e artífices), com 16.0\%.

Os participantes residiam maioritariamente na zona centro, nomeadamente nos distritos de Coimbra, Castelo Branco, Leiria, Guarda, Aveiro, Viseu, Santarém, Portalegre e Lisboa. Eram, na sua grande maioria, casados (93\%) e tinham em média dois filhos. Considerando as características do agregado familiar, a maioria das famílias era composta pelo casal e dois filhos (55\%), ou pelo casal e um filho (26\%).

No que respeita às crianças filhas dos participantes $(n=100), 51 \%$ eram do sexo feminino e $98 \%$ de nacionalidade portuguesa. A sua idade média foi de 8.69 anos $(D P=3.68)$, com um mínimo de 2 e máximo de 16 anos. Todas as crianças se encontravam a frequentar níveis de ensino correspondentes à sua faixa etária (do pré-escolar ao $11^{\circ}$ ano), com exceção de duas crianças (de dois e três anos) que ainda não estavam a frequentar nenhuma instituição de ensino. No que se refere às condições crónicas de saúde, 48 crianças apresentavam diagnóstico de diabetes 
mellitus tipo I, 44 de asma e oito de artrite idiopática juvenil. O tempo médio ocorrido após o diagnóstico foi de 4.87 anos $(D P=3.11)$.

Ao longo do processo de adaptação desta versão do CHIP, foram desenvolvidos alguns estudos exploratórios com este instrumento, prévios ao que será aqui apresentado. Cunha e Relvas (2009) compararam os dados originais fornecidos pelos autores, no que respeita à utilização dos três padrões de coping, com uma amostra portuguesa de 93 mães e pais de crianças com diferentes doenças crónicas (e.g., diabetes mellitus tipo I, asma, doença renal, doença hepática, entre outras). Os resultados revelaram valores significativamente mais altos nos Padrões I, II e III na amostra portuguesa, sugerindo uma tendência para estas mães e pais atribuírem maior importância (grau de utilidade) aos comportamentos de coping dos três padrões, quando comparados com a amostra estudada pelos autores. Um estudo exploratório de validação do CHIP foi realizado por Almeida (2013), no âmbito de uma investigação de mestrado integrado em Psicologia, apresentada à Faculdade de Psicologia e de Ciências das Educação da Universidade de Coimbra. Neste estudo, foi utilizada parte da amostra descrita no primeiro momento de recolha de dados e realizada uma análise fatorial exploratória do instrumento, que sugeriu uma estrutura fatorial diferente da proposta pelos autores (McCubbin et al., 1983).

\section{Estudos descritivos}

No Quadro 2 encontra-se apresentada uma análise descritiva dos 45 itens do CHIP, onde constam os valores para a média, desvio-padrão, moda, mínimo-máximo, assimetria e curtose.

Quadro 2.

Estatísticas descritivas dos itens do CHIP

\begin{tabular}{lcccccc}
\hline Item & $M$ & $D P$ & Moda & Mín-Máx & Assimetria & Curtose \\
\hline 1 & 2.61 & 0.70 & 3 & $0-3$ & -2.15 & 4.82 \\
2 & 1.99 & 0.93 & 2 & $0-3$ & -0.89 & 0.11 \\
3 & 2.71 & 0.59 & 3 & $0-3$ & -2.56 & 7.91 \\
4 & 2.07 & 1.08 & 3 & $0-3$ & -0.97 & -0.35 \\
\hline
\end{tabular}




\begin{tabular}{|c|c|c|c|c|c|c|}
\hline 5 & 2.66 & 0.53 & 3 & $1-3$ & -1.23 & 0.53 \\
\hline 6 & 2.60 & 0.71 & 3 & $0-3$ & -2.08 & 4.37 \\
\hline 7 & 1.94 & 1.01 & 2 & $0-3$ & -0.78 & -0.39 \\
\hline 8 & 2.01 & 0.90 & 2 & $0-3$ & -0.80 & 0.04 \\
\hline 9 & 1.24 & 1.01 & 2 & $0-3$ & 0.18 & -1.13 \\
\hline 10 & 2.34 & 0.71 & 3 & $0-3$ & -0.96 & 0.88 \\
\hline 11 & 2.13 & 0.90 & 2 & $0-3$ & -0.97 & 0.29 \\
\hline 12 & 1.79 & 1.05 & 2 & $0-3$ & -0.51 & -0.90 \\
\hline 13 & 1.59 & 0.97 & 2 & $0-3$ & -0.39 & -0.82 \\
\hline 14 & 1.86 & 1.00 & 2 & $0-3$ & -0.62 & -0.62 \\
\hline 15 & 2.69 & 0.49 & 3 & $1-3$ & -1.20 & 0.24 \\
\hline 16 & 2.60 & 0.52 & 3 & $1-3$ & -0.72 & -0.81 \\
\hline 17 & 2.23 & 0.71 & 2 & $0-3$ & -0.85 & 1.08 \\
\hline 18 & 2.20 & 0.89 & 3 & $0-3$ & -1.00 & 0.32 \\
\hline 19 & 2.30 & 0.71 & 2 & $0-3$ & -1.25 & 2.59 \\
\hline 20 & 2.51 & 0.63 & 3 & $0-3$ & -1.26 & 2.12 \\
\hline 21 & 2.42 & 0.69 & 3 & $0-3$ & -1.31 & 2.37 \\
\hline 22 & 1.76 & 0.81 & 2 & $0-3$ & -0.65 & 0.14 \\
\hline 23 & 2.67 & 0.49 & 3 & $1-3$ & -0.92 & -0.69 \\
\hline 24 & 2.07 & 0.77 & 2 & $0-3$ & -0.69 & 0.45 \\
\hline 25 & 2.22 & 0.71 & 2 & $0-3$ & -0.83 & 1.10 \\
\hline 26 & 2.01 & 0.65 & 2 & $0-3$ & -0.81 & 2.04 \\
\hline 27 & 2.19 & 0.79 & 2 & $0-3$ & -1.05 & 1.22 \\
\hline 28 & 2.21 & 0.77 & 2 & $0-3$ & -0.95 & 0.95 \\
\hline 29 & 1.69 & 0.99 & 2 & $0-3$ & -0.37 & -0.85 \\
\hline 30 & 1.69 & 0.94 & 2 & $0-3$ & -0.21 & -0.82 \\
\hline 31 & 2.52 & 0.66 & 3 & $0-3$ & -1.66 & 3.80 \\
\hline 32 & 1.89 & 0.88 & 2 & $0-3$ & -0.53 & -0.34 \\
\hline 33 & 1.66 & 0.90 & 2 & $0-3$ & -0.36 & -0.56 \\
\hline 34 & 1.85 & 0.89 & 2 & $0-3$ & -0.63 & -0.17 \\
\hline 35 & 2.71 & 0.53 & 3 & $0-3$ & -1.91 & 4.48 \\
\hline 36 & 2.53 & 0.76 & 3 & $0-3$ & -1.92 & 3.70 \\
\hline 37 & 1.18 & 1.05 & 0 & $0-3$ & 0.28 & -1.20 \\
\hline 38 & 2.30 & 0.79 & 3 & $0-3$ & -1.13 & 1.04 \\
\hline 39 & 1.62 & 0.96 & 2 & $0-3$ & -0.26 & -0.84 \\
\hline 40 & 2.29 & 0.76 & 2 & $0-3$ & -1.24 & 1.90 \\
\hline 41 & 2.71 & 0.51 & 3 & $0-3$ & -1.89 & 4.71 \\
\hline 42 & 1.22 & 1.02 & 0 & $0-3$ & 0.21 & -1.15 \\
\hline 43 & 2.51 & 0.68 & 3 & $0-3$ & -1.48 & 2.41 \\
\hline 44 & 2.51 & 0.74 & 3 & $0-3$ & -1.69 & 2.80 \\
\hline 45 & 2.72 & 0.51 & 3 & $0-3$ & -1.95 & 4.98 \\
\hline
\end{tabular}

O item 45 ("Fazer coisas com o meu filho") é o que apresenta uma média mais elevada $(M=2.72 ; D P=0.51)$, enquanto o item 37 ("Permitir a mim mesmo(a) zangar-me") obteve a média mais baixa $(M=1.18$; $D P=1.05)$. Com exceção dos itens 5, 15, 16 e 23, todos os restantes 
apresentam valores que oscilam entre 0 e 3 , sendo o valor mais frequente o 2 ("útil") Quanto à assimetria, atendendo ao constructo avaliado pelo CHIP, todos os itens apresentam um valor negativo com exceção dos itens 9, 37 e 42. Já para a curtose (grau de achatamento da distribuição), os itens que se encontram mais afastados do valor zero são os itens 1, 3, 6, 35, 41 e 45 .

Considerando as duas outras alternativas de resposta aos itens, aplicáveis quando os pais referem não utilizar o comportamento de coping ("não lido desta forma porque"), verificou-se que os itens $13(n=9), 37(n=9)$ e 39 ( $n=$ 9) foram os mais cotados na opção "decidi não fazê-lo" e que os itens 34 ( $n=$ 9), $13(n=7)$ e $29(n=7)$ foram os mais frequentes na opção "não é possível".

\section{Estudos de precisão: Consistência interna}

A evidência de precisão dos itens do CHIP foi averiguada através da análise da consistência interna, com recurso ao cálculo do coeficiente alfa de Cronbach para os três Padrões de Coping identificados nos estudos de análise fatorial exploratória (cf. Quadro 3). Assim sendo, o Fator 1 apresenta um valor de .88 , o Fator 2 de .82 e o Fator 3 de .80 , valores estes considerados como bons indicadores de fiabilidade, segundo Pestana e Gageiro (2008). De notar que qualquer um destes valores é superior aos obtidos na versão original do CHIP, que oscilavam entre .71 e .79. A análise dos valores do coeficiente alfa de Cronbach, para cada um dos padrões ao excluir qualquer um dos itens, indica-nos que a sua exclusão não aumenta de forma expressiva a consistência interna. Os valores das correlações item-total corrigidas revelam uma adequada capacidade discriminante de todos os itens $(r>.30)$, à exceção dos itens $31(r=.12)$ e $44(r=.28)$, com os valores a oscilarem entre .41-.64 para F1, .12-.60 para F2 e .28-.56 para F3.

\section{Estudos de precisão: Acordo entre informadores}

Para 39 crianças da amostra total em relação às quais foi preenchida uma versão do CHIP pelo pai e pela mãe (de forma independente), procedeu-se a 
um estudo de acordo entre informadores. Para tal, calcularam-se os coeficientes de correlação de Pearson entre os três padrões considerados nos estudos de análise fatorial exploratória para as cotações de pais e mães. Para os dois primeiros fatores obtiveram-se correlações moderadas e estatisticamente significativas: $\mathrm{F} 1=.44$ e F2 $=.54(p<.01)$. Quanto ao F3, a correlação obtida não alcançou o limiar de significância estatística $(r=.25, \mathrm{~ns})$.

\section{Estudos de validade de constructo: Análise fatorial exploratória}

A evidência de validade interna do CHIP foi verificada através de estudos de análise fatorial exploratória. Assim, num primeiro momento, foi averiguado o cumprimento dos pressupostos para a realização da referida análise. Em relação ao critério de Kaiser-Meyer-Olkin (KMO), o valor obtido de .769 revelou-se ajustado para a realização da análise em componentes principais, tal como o teste de esfericidade de Bartlett, $\chi^{2}(990)=2682.27, p<.001$, mostrou que existem correlações entre as variáveis (Pestana \& Gageiro, 2008).

A análise da matriz de componentes inicial não rodada apontava para uma solução de 12 fatores (com valores próprios superiores a 1) que, em conjunto, explicariam $67.12 \%$ da variância total. No entanto, a análise do scree-plot indicou que uma solução de três fatores seria plausível, com base no ponto de inflexão da curva (cf. Figura 1).

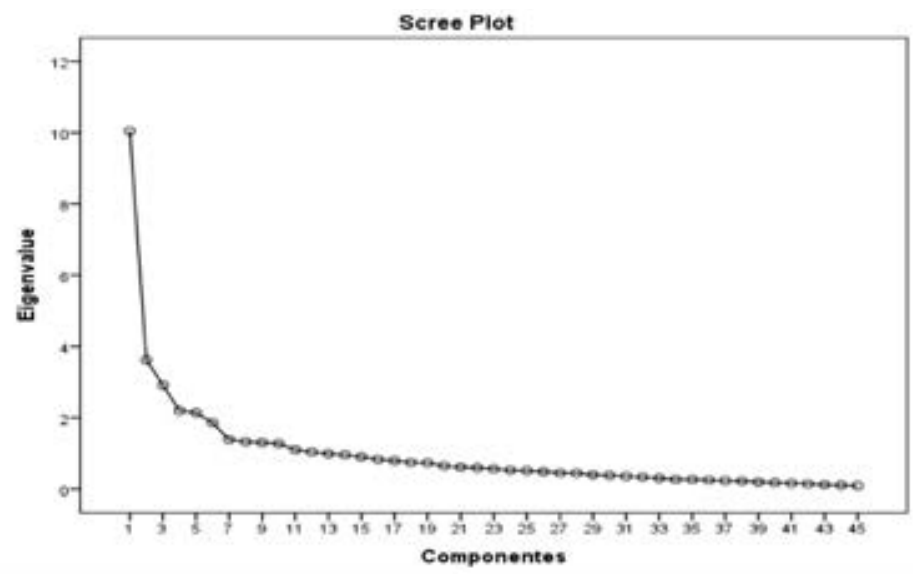

Figura 1. Scree-Plot: Solução inicial para os 45 itens do CHIP. 
Neste sentido, foi efetuada uma rotação varimax forçada a três fatores, permitindo assim comparar os resultados obtidos com a estrutura fatorial sugerida pelos autores do CHIP. A análise do Quadro 3 permite concluir que, apesar do recurso a uma solução trifatorial que explica $36.85 \%$ da variância total, semelhante aos três padrões de coping identificados por McCubbin et al. (1983), existem diversos itens que não se encontram agrupados nos mesmos fatores, não se confirmando assim uma correspondência com a escala original, em termos estatísticos e teóricos.

Assim, a primeiro fator, composto por 15 itens com saturações a oscilarem entre .454 e .677 , corresponde ao Padrão II identificado por McCubbin et al., (1983) - Manutenção do Suporte Social, Auto-Estima e Estabilidade Psicológica, com 14 itens comuns a ambas as soluções (todos os itens com exceção do item 28). O item 28 ("Dizer a mim próprio(a) que tenho muitas coisas que devo agradecer"), não obstante na versão original pertencer ao Padrão I, pode considerar-se teoricamente coerente com um comportamento de manutenção de estabilidade psicológica. Este fator reúne, assim, 15 itens que remetem essencialmente para comportamentos relacionados com a manutenção de auto-estima e estabilidade psicológica (cf. Quadro 3).

Como consta do Quadro 3, o segundo fator engloba 16 itens, com saturações entre .290 e .714, dos quais 11 itens pertencem ao Padrão I da versão original do CHIP - Integração Familiar, Cooperação e Definição Otimista da Situação (itens 1, 3, 8, 13, 18, 21, 26, 31, 36, 41 e 45). Note-se que os itens 8, 26 e 31 apresentam dupla saturação e que a sua inclusão neste fator se ficou a dever à sua consonância teórica com a versão original da escala. Os cinco itens restantes que integram este fator $(2,24$, 30, 33 e 34) pertencem originalmente aos outros dois padrões. Assim, os itens 2, 24 e 33 (originalmente pertencentes aos Padrão II), teoricamente coerentes com a noção de suporte social, passam a integrar este fator. Também o item 34 ("Sair com o meu cônjuge/companheiro(a) de forma regular"), apesar de originalmente pertencente ao Padrão II, apresenta coerência teórica com a noção de fortalecimento das relações familiares, daí a sua fixação neste padrão. Por fim, originalmente pertencente ao Padrão III, o item 30 ("Explicar a situação familiar a amigos e vizinhos 
para que eles nos compreendam"), pode considerar-se representativo de um comportamento consonante com a noção de manutenção de suporte social (apesar do menor valor de saturação neste padrão). Este fator reúne, assim, 16 itens que remetem essencialmente para comportamentos de fortalecimento da vida familiar e manutenção de suporte social.

Por fim, encontramos o terceiro fator com 14 itens, com saturações a assumirem valores entre .244 e .689, com um total de sete itens (itens 5, $10,15,20,25,35,40)$ que pertencem ao Padrão III do CHIP - Compreensão da Situação Médica através da Comunicação com outros Pais e Consulta com os Membros da Equipa de Saúde (cf. Quadro 3). Os restantes itens que integram este fator pertencem originalmente ao Padrão I, não obstante alguns estarem centrados na situação/cuidados médicos (itens 11, 43). Os itens 6, 16, 23 e 44 contêm afirmações relacionadas com a avaliação que os pais fazem da condição e cuidados médicos prestados à criança e são tradutoras de uma definição otimista da situação (e.g., item 6 "Acreditar que o meu filho vai melhorar"). Este fator reúne, assim, itens que remetem para a compreensão da situação médica através do relacionamento com outros pais e com a equipa de saúde e para uma definição otimista da situação. Excetua-se o item 38 ("Investir tudo de mim no(s) meu(s) filho(s)") que, teoricamente, não se enquadra nesta composição.

Quadro 3.

Matriz rodada, comunalidades e variância explicada (Rotação Varimax) - CHIP

\begin{tabular}{|c|c|c|c|c|}
\hline \multirow[t]{2}{*}{ Itens } & \multicolumn{3}{|c|}{ Fatores } & \multirow{2}{*}{$b^{2}$} \\
\hline & 1 & 2 & 3 & \\
\hline 42. Poder afastar-me das tarefas... & .677 & & & .458 \\
\hline 27.Tornar-me mais auto-confiante... & .633 & & & .401 \\
\hline 14. Dar um tempo... & .610 & & & .372 \\
\hline 32. Manter-me em forma... & .604 & & & .365 \\
\hline 4. Dormir... & .598 & & & .358 \\
\hline 12. Comer... & .571 & & & .326 \\
\hline 28. Dizer a mim próprio(a) que tenho... & .565 & & & .319 \\
\hline 22. Investir tempo e energia... & .558 & & & .311 \\
\hline 19. Desenvolver-me enquanto... & .556 & & & .309 \\
\hline 37. Permitir a mim mesmo(a)... & .547 & & & .299 \\
\hline 39. Falar com alguém... & .546 & & & .298 \\
\hline
\end{tabular}


7. Trabalhar, ter um...

26. Fazer coisas com outros...

.500 .461

9. Comprar presentes para...

.492

.459

.411

29. Concentrar-me em...

$.483 \quad .438$

.425

17. Estabelecer relações...

$.454 \quad .434$

.394

8. Mostrar que...

$.370 \quad .310$

.233

34. Sair com o meu cônjuge...

.714

.510

1. Conversar, com o cônjuge...

.630

.316

.497

24. Receber amigos...

.617

.381

33. Envolver-me em atividades...

.401

.596

.516

3. Confiar no meu cônjuge...

.592

.350

2. Estabelecer relacionamentos...

.570

.325

36. Fortalecer a relação com...

.542

.294

45. Fazer coisas com...

.529

.331

.390

21. Fazer coisas enquanto...

.525

.276

13. Pedir ajuda a outros membros...

.429

.184

41. Tentar manter a estabilidade...

.412

.337

.284

18. Acreditar ...

.294

.086

30. Explicar a situação familiar a...

.290 .084

15. Falar com o médico sobre...

\begin{tabular}{lll} 
& .689 & .475 \\
& .650 & .423 \\
& .639 & .408 \\
& .607 & .368 \\
& .546 & .298 \\
.324 & .545 & .402 \\
& .490 & .240 \\
& .488 & .238 \\
& .487 & .237 \\
& .487 & .237 \\
.349 & .454 & .328 \\
& .423 & .179 \\
& .417 & .174 \\
& .376 & .141 \\
& .244 & .060 \\
11.77 & 10.97 & \\
\hline
\end{tabular}

5. Falar com o pessoal médico...

16. Acreditar que o centro de saúde/hospital...

35. Ter a certeza que os tratamentos...

43. Saber que o meu filho é visto...

25. Ler sobre a forma como...

40. Ler mais sobre o problema...

11. Tomar conta de todo o equipamento...

20. Falar com outros pais...

23. Acreditar que o meu filho...

31. Encorajar a criança...

38. Investir tudo de mim...

10. Falar com outras pessoas/pais...

6. Acreditar que o meu filho...

.060

44. Acreditar que as coisas...

$14.11 \quad 11.77 \quad 10.97$

\% Variância explicada

ores a .30 . A negrito encontram-se assinaladas as saturações dos itens com dupla saturação incluídos noutro padrão. 
Conclui-se assim que a solução obtida com base nos estudos de análise fatorial exploratória apresenta uma sobreposição com a versão original do CHIP para 32 dos 45 itens.

Estudos de validade de constructo: Análise fatorial confirmatória

Foram realizados estudos de análise fatorial confirmatória do modelo proposto pelos autores da versão original do CHIP de modo a analisar a possibilidade da sua adequação para os nossos dados $(N=125)$. Deste modo, foi testado um modelo composto por três fatores: Padrão I (itens $1,3,6,8,11,13,16,18,21,23,26,28,31,36,38,41,43,44,45)$, Padrão II (itens 2, 4, 7, 9, 12, 14, 17, 19, 22, 24, 27, 29, 32, 33, 34, 37, 39, 42) e Padrão III (itens 5, 10, 15, 20, 25, 30, 35, 40). Apesar de todas as saturações estandardizadas se situarem acima de .30: Padrão $I=.324-.635$, Padrão II $=.402-.710$ e Padrão III $=.301-.637$, os índices de ajustamento do modelo testado revelaram-se inadequados. Assim, $\chi^{2}=1896.80(p<$ $.001), \chi^{2} / g l=2.011$, Comparative Fit Index $=.543$, Goodness of Fit Index $=.590$, sendo estes dois últimos valores muito distantes dos valores de referência (.95). O mesmo se passa com o Root Mean Square Error of Approximation com um valor de .090 ( Lo $=.084, \mathrm{Hi}=.096)$, também acima do .06 recomendado na literatura (Byrne, 2010; Marôco, 2010). Mesmo atendendo aos índices de modificação, os índices de ajustamento não alcançam os valores esperados. Conclui-se, desta forma, que apesar das discrepâncias encontradas em relação à composição dos três padrões aquando dos estudos de análise fatorial exploratória, também o modelo original do CHIP não se adequa aos dados recolhidos.

\section{Estudos de validade: Comparação de grupos}

Foi ainda efetuado um estudo com recurso ao teste $t$ de Student para amostras independentes, no sentido de comparar os resultados obtidos nos três padrões considerados, nos grupos de pais e mães de crianças com 
asma ( $n=58)$ e diabetes $(n=63)$. O grupo de pais e mães de crianças com artrite juvenil não foi considerado para este estudo, atendendo à sua reduzida dimensão $(n=14)$.

Quadro 4

Comparação de grupos: Teste $t$ de Student asma e diabetes

\begin{tabular}{lcccccc}
\hline $\begin{array}{l}\text { Padrão } \\
\text { CHIP }\end{array}$ & \multicolumn{2}{c}{$\begin{array}{c}\text { Grupo Asma } \\
(n=58)\end{array}$} & \multicolumn{2}{c}{$\begin{array}{c}\text { Grupo Diabetes } \\
(n=63)\end{array}$} \\
\hline & $M$ & $D P$ & $M$ & $D P$ & $t$ & $p$ \\
F1 & 26.62 & 9.46 & 28.79 & 7.58 & -1.399 & .164 \\
F2 & 34.24 & 7.29 & 36.15 & 6.05 & -1.589 & .115 \\
F3 & 34.56 & 5.27 & 35.16 & 4.68 & -0.665 & .507
\end{tabular}

Nota. F1, F2 e F3 = Fatores identificados nos estudos de AFE.

Como se observa no Quadro 4, apesar do grupo de pais e mães de crianças com diabetes apresentar uma média tendencialmente superior ao grupo de pais e mães de crianças com asma para os três padrões do CHIP, nenhuma diferença alcança o limiar de significância estatística.

\section{Aplicação}

\section{Como aplicar, cotar e interpretar?}

O CHIP foi desenvolvido para avaliar a perceção que os pais têm sobre os comportamentos de coping que utilizam e consideram uteis (ou não) para lidar com a vida familiar quando têm um filho gravemente doente, por um curto período de tempo, ou com uma condição médica crónica que exige cuidados continuado e/ou cronicamente doente. Como tal, poderá ser aplicado a pais (ou outros cuidadores) de crianças nestas situações.

Trata-se de instrumento de auto-resposta, de aplicação individual, e para o seu preenchimento é apenas necessária uma versão em papel e um lápis/caneta. Ao respondente é solicitado que classifique, numa escala de 0 a 3 (Nada útil; Minimamente Útil; Útil; Extremamente útil), a utilidade, para lidar com a situação familiar, de cada um dos 45 comportamentos 
apresentados. Se um comportamento de coping não for utilizado, os respondentes deverão indicar porque razões não o utilizam: a) porque decidi não fazê-lo; ou b) porque não é possível.

No que respeita aos procedimentos de cotação, poderá ser calculado um resultado total do CHIP, através da soma dos valores correspondentes aos níveis da escala ( 0 = Nada útil; 1 = Minimamente Útil; 2 = Útil; 3 = Extremamente útil), nos 45 itens. Os comportamentos que não são utilizados são equivalentes a 0 . Podem ainda ser calculados resultados para cada padrão de coping, somando as pontuações dos itens correspondentes. Considerando a estrutura fatorial apresentada neste estudo, o Padrão I integra 15 itens $(4,7,9,12,14,17,19,22,27,28,29,32,37$, 39, 42), o Padrão II engloba 16 itens (1, 2, 3, 8, 13, 18, 21, 24, 26, 30, $31,33,34,36,41,45)$ e o Padrão III inclui os restantes 14 itens do instrumento $(5,6,10,11,15,16,20,23,25,35,38,40,43,44)$.

\section{Vantagens, limitações e estudos futuros}

Desenvolvido para avaliar a perceção dos pais sobre os comportamentos de coping que utilizam para lidar com a vida familiar quando têm um filho gravemente doente, o CHIP revela-se um instrumento útil para investigação, apresentando ainda potencialidades enquanto ferramenta clínica, podendo auxiliar os profissionais no contacto com famílias de crianças com doenças graves/crónicas e a perceber como cada pai está a lidar com a situação (McCubbin, Thompson, \& McCubbin, 2001).

A versão portuguesa do CHIP apresenta uma estrutura fatorial semelhante à sua versão original, no que se refere ao número de fatores. No entanto, os fatores extraídos revelam uma configuração distinta, destacando-se neste estudo: a) a composição de um fator que inclui itens que traduzem tentativas de envolvimento em atividades que aumentam a autoestima e comportamentos para lidar com as tensões e pressões psicológicas (Padrão I); b) a agregação de itens que traduzem os esforços parentais para desenvolver relacionamentos com outras pessoas com 
comportamentos focalizados no fortalecimento da vida familiar (Padrão II); e c) a integração conjunta de itens relacionados com a compreensão da situação médica e a avaliação que os pais fazem da situação (Padrão III). Esta versão revela boas qualidades psicométricas, com valores de consistência interna nos três padrões de coping superiores aos da versão original. Não sendo o CHIP um instrumento aplicável a nenhuma doença particular, este estudo vem também sugerir a ausência de diferenças na utilização de comportamentos de coping entre pais de crianças com asma e com diabetes.

Uma limitação a apontar neste estudo, particularmente na interpretação dos resultados relativos à estrutura fatorial do instrumento, prende-se com o reduzido tamanho da amostra utilizada. Deste modo, estudos futuros de validação beneficiariam com a integração de amostras mais alargadas, incluindo diferentes parâmetros, como outros diagnósticos, características da doença (e.g., duração e gravidade) ou diferentes grupos etários. A consideração futura de outras medidas de avaliação (e.g., coping parental, funcionamento familiar) poderá, igualmente, representar um contributo para o estudo da evidência de validade deste instrumento.

\section{Bibliografia}

Aguilar-Vafaie, M.A. (2008). Coping-Health Inventory for Parents: Assessing coping among Iranian parents in the care of children with cancer and introductory development of an adapted Iranian Coping-Health Inventory for Parents. Children's Health Care, 37, 237-260. doi: 10.1080/02739610802437202

Almeida, A. R. (2013). Coping parental e resistência familiar na asma e na diabetes: Um estudo exploratório. Dissertação de Mestrado não publicada. Faculdade de Psicologia e de Ciências da Educação. Universidade de Coimbra.

Auslander, W., Bubb, J., Rogge, M., \& Santiago, J. (1993). Family stress and resources: Potential. areas of intervention on children recently diagnosed with diabetes. Health $\&$ Social Work, 18(2), 101-113. doi: 10.1093/hsw/18.2.101

Byrne, B. M. (2010). Structural equation modelling with AMOS: Basic concepts, applications, and programming (2nd ed.). New York: Routledge.

Cavallo, S., Feldman, D. E., Swaine, B., Meshefedjian, G., Malleson, P. N., \& Duffy, C. M. (2009). Is parental coping associated with quality of life in juvenile idiopathic arthritis? Pediatric Rheumatology, 7, 7. doi: 10.1186/1546-0096-7-7

Cunha, A. I. (2011). Histórias e trajectórias de adaptação e resiliência familiar na doença crónica pediátrica. Dissertação de Doutoramento não publicada. Faculdade de Psicologia 
e de Ciências da Educação. Universidade de Coimbra. Disponível em: http://hdl.handle. net/10316/20310

Cunha, A. I. \& Relvas, A. P. (2009). Estratégias de coping parental na doença pediátrica: Dados preliminares sobre a utilização do CHIP numa amostra portuguesa. Poster apresentado no I Congresso Luso-Brasileiro de Psicologia da Saúde (Faro, Portugal.).

Garro, A. (2011). Coping patterns in Latino families of children with asthma. Journal of Pediatric Health Care, 25(6), 347-354. doi: 10.1016/j.pedhc.2010.04.005

Goldbeck, L. (2001). Parental coping with the diagnosis of childhood cancer: Gender effects, dissimilarity within couples, and quality of life. Psycho-Oncology, 10, 325-335. doi: 10.1002/pon.530

Han, H., Cho, E. J., Kim, D., \& Kim, J. (2009). The report of coping strategies and psychosocial adjustment in Korean mothers of children with cancer. Psycho-Oncology, 18, 956-954. doi: 10.1002/pon.1514

Hawley, D. R., \& DeHaan, L. (1996). Toward a definition of family resilience: Integrating life-span and family perspectives. Family Process, 35, 283-298. doi: 10.1111/ j.1545-5300.1996.00283.x

Instituto Nacional de Estatística (2010). Classificação portuguesa das profissões 2010. Lisboa: INE.

Lesar, S., \& Maldonado, Y. (1996). Parental coping strategies in families of HIV-infected children. Children's Health Care, 25(1), 19-35. doi: 10.1207/s15326888chc2501_3

Marôco, J. (2010). Análise de equações estruturais: Fundamentos teóricos, software $\mathcal{E}$ aplicações. Pêro Pinheiro: ReportNumber.

McCubbin, H. I., McCubbin, M. A., Nevin, R., \& Cauble, E. (1981). Coping Health Inventory for Parents (CHIP). In H. I. McCubbin, A. I. Thompson, \& M. A. McCubbin (2001). Family assessment: Resiliency, coping and adaptation-inventories for research and practice (pp. 407-453). Madison: University of Wisconsin System.

McCubbin, H. I., McCubbin, M. A., Patterson, J. M., Cauble, A. E., Wilson, L. R., \& Warwick, W. (1983). CHIP-Coping Health Inventory for Parents: An assessment of parental coping patterns in the care of the chronically ill child. Journal of Marriage and the Family, 45 (2), 359-370. doi: 10.2307/351514

McCubbin, H. I., Thompson, A. I., \& McCubbin, M. A. (2001). Family assessment: Resiliency, coping and adaptation- Inventories for research and practice. Madison: University of Wisconsin System.

McCubbin, M. A. \& McCubbin, H. I. (1996). Resiliency in families: A conceptual. model of family adjustment and adaptation in response to stress and crises. In H. I. McCubbin, A. I. Thompson, \& M. A. McCubbin (2001). Family assessment: Resiliency, coping and adaptation-Inventories for research and practice. (pp. 1-64). Madison: University of Wisconsin System.

$\mathrm{Mu}$, P. (2005). Paternal reactions to a child with epilepsy: Uncertainty, coping strategies, and depression. Journal of Advanced Nursing, 49(4), 367-376. doi: 10.1111/ j.1365-2648.2004.03300.x

Patterson, J. M., Budd, J., Goetz, D., \& Warwick, W. J. (1993). Family correlates of a 10-year pulmonary health trend in cystic fibrosis. Pediatrics, 91(2), 383-389.

Pereira, M. G., Almeida, C., Rocha, L., \& Leandro, E. (2011). Predictors of adherence, metabolic control and quality of life in adolescents with Type 1 Diabetes. In L. Chih-Pin (Ed.). Type 1 Diabetes: Complications, pathogenesis and alternative treatments (Book 3) (pp. 119-140). USA: InTech.

Pestana, M. H., \& Gageiro, J. N. (2008). Análise de dados para ciências sociais: a complementaridade do SPSS ( $5^{\mathrm{a}}$ ed.). Lisboa: Sílabo. 
Stallwood, L. (2005). Influence of caregiver stress and coping on glygemic control of young children with diabetes. Journal of Pediatric Health Care, 19, 293-300. doi: 10.1016/j. pedhc.2005.04.003

Walsh, F. (1998). Strengthening family resilience. New York: The Guilford Press.

Walsh, F. (2002). A family resilience framework: Innovative practice applications. Family Relations, 51(2), 130-137. doi: 10.1111/j.1741-3729.2002.00130.x 